\title{
Research on the Optimization Model of Energy Efficiency of Industrial Park
}

\author{
$\mathrm{He} \mathrm{Xin}^{*}$ and Wang Zhenquan
}

School of Economics \& Management, Beijing Institute of Petrochemical Technology, Beijing 102617, China

\begin{abstract}
In order to take the reduction of pollution emissions as the target, this paper generates the model parameters based on the input-output relationship between material flow and energy flow of regional inter-industry, and builds the model of energy efficiency optimization of industrial park with the distribution of regional industry structure as the control variable. The modeling process is discussed in detail in model structure, elements and optimization objective and
\end{abstract} control variable etc. and the suggestions on further research are given.

Keywords: Energy efficiency, industrial park, modeling, optimization model.

\section{INTRODUCTION}

As an area marked out through administrative measure by the government of a country or a region, the industrial park is a modern production area which gathers various production factors, scientifically integrates the resources, and optimizes the functional layout. The problems on the evaluation and modeling of the supply and need, utilization efficiency of energy resources are concerned by some scholars, and many researches on the construction, development, planning and evaluation, etc. of eco-industrial park appear [1-4]. Nevertheless, different from the administrative region, the industrial park has no specific centralized department to conduct statistics to the energy consumption and collect and manage the statistic data, which is the main difficulty of building the optimization model of energy efficiency of industrial park. This paper theoretically discusses the building process of the optimization model of energy efficiency of industrial park, and presents some relevant suggestions.

\section{MODELING PRINCIPLE AND FUNDAMENTAL ASSUMPTION}

\subsection{Optimization Objective and Modeling Framework}

The evaluation criterion of energy efficiency mainly covers three aspects, namely living, resources and environment. The main objective of the energy efficiency optimization of industrial park is to save resources as far as possible and protect the environment on condition of guaranteeing certain benefit of enterprise [5].

Under certain technical condition, some by-products generated from production and processing can directly substitute for raw material, or directly used for final

*Address correspondence to this author at the School of Economics \& Management, Beijing Institute of Petrochemical Technology, Beijing 102617, China; Tel: 010-52489515; Fax: 010-58850501-1987;

E-mail: hexin@bipt.edu.cn consumption; some can generate new recyclable by-products or by-products which cannot be recycled after being processed. Under the existing technical condition, the byproducts that cannot be recycled are likely to cause certain influence on the environment through emission, and such byproduct is called as pollutant. In this way, the production of single product can be boiled down to the input-output process of raw material (energy) - product. In which, the recycle utilization of by-product is a key to improve its energy efficiency. The more the emitted by-products, the lower the energy efficiency, and the positive relationship between by-product utilization ratio and energy efficiency indicates that the improvement of by-product utilization ratio is the effective way to improve the comprehensive energy efficiency of the park, as shown in Fig. (1).

Therefore, the specific objective of energy efficiency optimization of industrial park can be identified as the improvement of by-product utilization ratio on condition of guaranteeing the gross output of the park; the general modeling framework can be designed to build an energy optimization model with the production process as center, including material flow conversion production module, energy conversion flow energy module and pollutant emission environment module $[6,7]$.

\subsection{Fundamental Assumption}

In order to be convenient for describing the model, it is necessary to perform fundamental assumption to the production structure of industrial park.

Assume that there is a sole corresponding relation between product and enterprise, namely that an enterprise only manufactures a kind of product, and a kind of main product is only manufactured by an enterprise in the park. Although a single enterprise in a park often manufactures multiple products, and a same kind of product is often manufactured by many enterprises, this simplified processing does not affect the description of the production structure in the park. In fact, the objective of business 


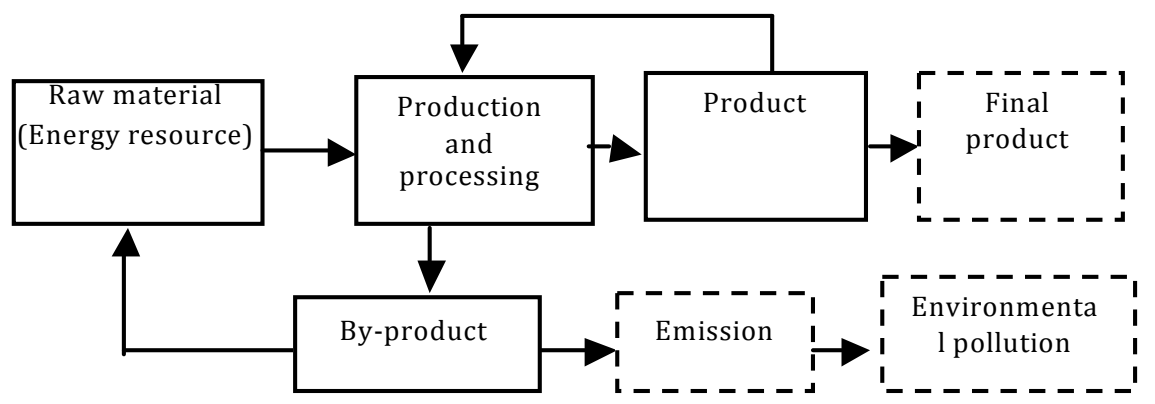

Fig. (1). Vector flow graph of single product production.

operation is economic accounting and enterprise development, and the energy optimization is to improve the energy efficiency of the whole park, which does not involve the problems such economic accounting, and has no conflict with the objective of improving the profit of enterprise.

In the survey period (also called as report period, it generally takes fiscal year as unit, and statistics should be conducted in investigation period if there is no special statement below), the total production quantity of a product is called as the gross output of this product, consisting of intermediate use, final consumption and imports from other places (imports for short), etc. Of which, "intermediate use" refers to the product used for production input, and the final consumption includes enterprise consumption of the park, as well as the product exported to the outside of the park through market (exports for short).

From Fig. (1), we can see that the production input mainly includes raw material and energy, which can be divided into initial input and recycled resource re-input, excluding the product input. Actually, the production purpose is to obtain the final product, while the product input occupies the final product, therefore, on the basis of the input-output principle of production, for the production module, it is assumed as follows:

1) For the given product, its production technology remains unchanged, namely that the production technology is exogenous,

2) What is reckoned in the final consumption must be the product,

3) The product and by-product entered in the production process are not reckoned in the initial input.

The imports may be the material which is same as local product, also may be the resource different from local product, and all of them are called as imported product. The production of it does not occupy local resource, nor pollute local environment. Therefore, for the imported product, it is assumed as follows:

1) The imported product is reckoned in intermediate use or final consumption, not reckoned in the initial input;

2) Only the initial input of the imported resource is reckoned, and the part used for the final consumption is not reckoned.

For the processing of by-product, it is assumed as follows:
1) The by-product substituted for the raw material to participate in the production enters into the production process for the second time, which is reckoned in the initial input, and correspondingly the replaced raw material should be deducted.

2) The by-product (such as industrial hot water and coal gas) substituted for the existing product and directly used for the final consumption is directly reckoned in the output of replaced product since it does not enter into the production process for the second time;

3) The by-product which cannot substitute for raw material and the existing product and is directly reckoned in the final consumption may be exported to the outside of the park, or directly consumed locally. Because it does not enter into the production process for the second time, it cannot be directly reckoned in the production input, and the processing method of it is to set a virtual production unit and reckon this byproduct in its final consumption;

4) The by-product which cannot be used entirely is reckoned in the pollutant according to the pollution level of its emission to the environment.

\section{MODEL STRUCTURE AND ELEMENTS}

\subsection{Main Product and Production Module}

Assume that there are $n$ enterprises in the park which manufacture $n$ kinds of products (main product), and the total output $x_{k}$ of No. $k$ product is called as gross output, and its distribution is shown as follows:

$x_{k}=\sum_{j=1}^{n} x_{k j}+y_{k}-z_{k},(k \in \underline{n})^{1}$

$x_{k j}$ is the quantity of No. $k$ product consumed to manufacture No. $j$ product, and the constituted matrix is called as intermediate use; $y_{k}$ is the quantity of products supplied to the market by the park, including final consumption or final product; $z_{k}$ is the quantity of this product imported (through market) from the outside of the park;

If $x_{k}=0$, this park does not manufacture this kind of product, and its consumption and intermediate use entirely

${ }^{1} n$ denotes set $\{1,2, \cdots, n\}$, the same below. 
depends on the import from the outside of the park, now $\sum_{j=1}^{n} x_{k j}+y_{k}=z_{k}$, indicating that this commodity should be deleted from the product list, thus $x_{k}>0$.

Denote the quantity of No. $k$ product directly consumed to manufacture unit $j$ product as $a_{k j}=x_{k j} / x_{j}$, which is called as direct consumption coefficient, and $\mathbf{A}=\left(a_{k j}\right)_{n \times n}$ is called as direct consumption coefficient matrix. Hence the vector formula of Formula (1) is:

$Y=(\mathbf{I}-\mathbf{A}) X+Z$

The above formula describes the composition of product's final consumption, which can de rewritten as:

$X=(\mathbf{I}-\mathbf{A})^{-1}(Y-Z)$

\subsection{Initial Input and Energy Module}

For clarity, the energy in the park only refers to primary energy, coal gas, electricity and petrochemicals used as fuel (such as steam, diesel and fuel oil), and all these are called as narrow energy; and the pressure gas, cold and hot source, etc are called as broad energy. Assume that the first $n_{1}$ enterprises in the park manufacture narrow energy and the following $n_{2}$ enterprises manufacture broad energy, and assume that the enterprises manufactured the energy are professional.

Assume that there are $r$ kinds of initial inputs in the park, divided into two resources, namely energy and nonenergy, of which, the first $s$ kinds are narrow energy. If we leave out the non-energy resource initially input and the energy which has been consumed before importing the product, the energy consumption of the park is the gross energy contained in initially input energy substance [8].

Denote No. $i$ Initial input material required by No. $k$ unit product as $t_{i k}$, and denote $\mathbf{T}=\left(t_{i k}\right)_{r \times n}$, which is called as resource consumption coefficient matrix. On condition of not replacing initial input with by-product, the initial input quantity of No. $i$ resource is $u_{i}=\sum_{k=1}^{n} t_{i k} x_{k},(i \in \underline{r})$, and the corresponding initial input vector is $U_{r}=\mathbf{T} X$. However, the equivalent by-product utilization quantity should be deducted from actual input quantity. Denote the energy vector of initial input resource as $\eta$, leave out the energy consumed in the early initial input, and the following $r-s$ vectors are 0 , simplified as $\eta \mathrm{s}$.

\subsection{By-Product and Environment Module}

Assume that $m$ kinds of by-products in all are manufactured during the production process of $n$ kinds of main products. If we denote the quantity of No. $j$ byproduct generated from manufacturing unit $k$ product as $b_{k j}$ and $\mathbf{B}=\left(b_{k j}\right)_{n \times m}$ is called as by-product production matrix, the total quantity of No. $j$ by-product generated by the given production structure is $b_{j}=\sum_{k=1}^{n} b_{k j} x_{k}(j \in \underline{m})$, and the vector of by-product in the park is $b=\left(b_{1}, \cdots, b_{m}\right)^{T}=\mathbf{B}^{T} X$.

The by-product recycle utilization is realized through replacing the initial input, but the possible scheme of replacement and its result are complicated. Based on the above fundamental assumption, the last scheme can be boiled down to direct substitution. The indirect substitution is obscure, therefore, only the direct substitution is taken into account in the model.

For the production of product $k$, if No. $j$ by-product can substitute for No. $i$ initial input, denote the quantity of No. $j$ by-product equivalent to No. $i$ unit initial input as $h_{i j}^{k}$, thus the initial input substitution coefficient matrix of the by-product substitution realized through the production of product $k$ is $\mathbf{H}_{k}=\left(h_{i j}^{k}\right)_{r \times m},(k \in \underline{n})$. If it cannot be replaced, make $h_{i j}^{k}=0$.

No. $i$ initial input required by the production of unit $k$ product is $t_{i k}$, and the equivalent No. $j$ by-product is $d_{j k}^{i} \triangleq t_{i k} h_{i j}^{k} \quad(k \in \underline{n}, i \in \underline{r}, j \in \underline{m})$. Hence the quantity of maximum by-product $j$ used by the production of unit $k$ product is $d_{j k} \triangleq \sum_{i=1}^{r} t_{i k} h_{i j}^{k}$. Call $\mathbf{D}=\left(d_{j k}\right)_{m \times n}$ as the byproduct consumption coefficient matrix, its No. $k$ column vector is:

$D_{k}=\mathbf{H}_{k}^{T} T_{k},(k \in \underline{n})$

Of which, $T_{k}$ is No. $k$ column of resource consumption coefficient matrix $T_{r \times n}$. For the given product structure $X$, the maximum consumption vector of No. $j$ by-product in the park is $\hat{d}_{j}=\sum_{k=1}^{n} d_{j k} x_{k}$, and denote $d_{\max }=\left(\hat{d}_{1}, \cdots, \hat{d}_{m}\right)^{T}=\mathbf{D} X$.

Because the substitution coefficient matrix has 3 groups of subscripts, it cannot be expressed with two-dimensional matrix tool. For this, we expand the matrix expression tool.

A matrix is a two-dimensional "row-column" form, and $\left\{\mathbf{H}_{k}: k \in \underline{n}\right\}$ in fact is $n$ sheets of $r \times m$ forms. Take its subscript as the third dimension, called as $k$ dimension, and the subscripts of each element of single $\mathbf{H}_{k}$ are respectively taken as $i$ dimension and $j$ dimension, hence the relational formula can be expressed with Fig. (2). In Fig. (2), matrix $\mathbf{T}=\left(t_{i k}\right)$ is at $i-k$ plane, $\mathbf{D}=\left(d_{j k}\right)$ is at $j-k$ plane, $\mathbf{H}_{1}$ is at $i-j$ plane of $k=1$, and $\mathbf{H}_{n}$ is at $i-j$ plane of $k=n$. The calculation of Formula (4) $D_{k}=\mathbf{H}_{k}^{T} T_{k},(k \in \underline{n})$ is carried out at corresponding $i-j$ plane, and the obtained vector $D_{k}$ constitutes matrix $\mathbf{D}=\left(d_{j k}\right)$ of $j-k$ plane. 
Table 1. Input-output table of energy- environment load of industrial park.

\begin{tabular}{|c|c|c|c|c|c|c|c|}
\hline \multicolumn{2}{|c|}{ Output } & \multirow{2}{*}{ Intermediate Use } & \multirow{2}{*}{ Final Product } & \multirow{2}{*}{ Imports } & \multirow{2}{*}{ Gross Output } & \multirow{2}{*}{ By-Product } & \multirow{2}{*}{ Relational Formula } \\
\hline Input & Dept. & & & & & & \\
\hline \multirow{2}{*}{ Product } & Energy & \multirow{2}{*}{$\mathrm{A}_{n \backslash n}$} & \multirow{2}{*}{$Y_{n}$} & \multirow{2}{*}{$Z_{n}$} & \multirow{2}{*}{$X=X_{D}+X_{T}$} & \multirow{2}{*}{$\mathrm{B}_{\text {IX } m}$} & \multirow{2}{*}{$Y=(\mathbf{I}-\mathbf{A}) X+Z$} \\
\hline & Non-energy & & & & & & \\
\hline \multirow{2}{*}{ Recycled product } & Energy & \multirow{2}{*}{$\mathrm{D}_{\pi \times n}$} & \multirow{2}{*}{ - } & \multirow{2}{*}{$d=\mathbf{D} X_{D}$} & \multirow{2}{*}{ Contamination coefficient $\delta$} & \multirow{2}{*}{ - } & \multirow{2}{*}{$b=\mathbf{B}^{T} X \quad d_{\max }=\mathbf{D} X$} \\
\hline & Non-energy & & & & & & \\
\hline \multirow{2}{*}{ Initial input } & Energy & \multirow{2}{*}{$\mathrm{T}_{r^{\prime} \mathbf{n}^{n}}$} & $\eta_{s}$ & \multirow{2}{*}{$u=\mathbf{T} X_{T}$} & \multirow{2}{*}{ - } & \multirow{2}{*}{$\mathbf{H}_{k}=\left(h_{i j}^{k}\right)_{r \times m}$} & \multirow{2}{*}{ 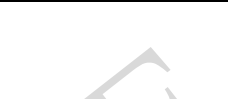 } \\
\hline & Non-energy & & - & & & & \\
\hline
\end{tabular}

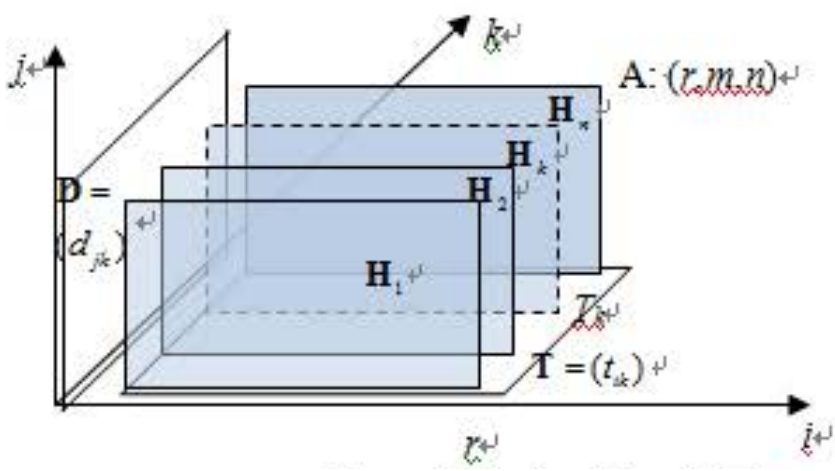

Fig. (2). Schematic diagram of three-dimensional matrix operation.

The expression of content and symbol of the above three modules can be boiled down to the input-output relationship as shown in Table 1, of which, all subscripts express the dimensions of vectors or matrixes, and the superscript " $T$ "expresses the transposition of matrix or vector, and $d$ is the actual usage amount of by-product.

\section{MODEL BUILDING}

\subsection{Constraint Conditions}

In order to use the parameters and variables in Table 1 to build the optimization model, it is necessary to further define the structure of the parameters or variables.

The parameter determined by production technology is called as the parameter of production technology, and the parameter only relates to the industrial structure of the park is called as the parameter of industrial structure; the variable changed with the market demand is called as market variable, and the parameter only determined by the nature of material is called as the parameter of material nature. In this way, the sizes of $\mathrm{A}_{n_{\times} n}, \mathrm{~B}_{n_{\times} m}, \mathrm{D}_{m_{\times^{n}}}$ and $\mathrm{T}_{r_{\times}}$and other parameters in Table $\mathbf{1}$ are determined by the process technology, but the contents are determined by the industrial structure of the park. Assume that the production specifications of the park remain unchanged, they are the parameters of industrial structure. Energy conversion coefficient $\eta_{s}$ and contamination coefficient $\delta_{m}$ are the parameter of material nature. The attribute of $\mathbf{H}_{k}=\left(h_{i j}^{k}\right)_{r \times m}$ relates to the product structure and material nature.
The final consumption $Y$ of product is market variable, and $X=(\mathbf{I}-\mathbf{A})^{-1}(Y-Z)$ is endogenous variable. Assume that the demand vector of the market to the product is $Y_{d}$, we obtain the first constraint condition:

$(\mathbf{I}-\mathbf{A}) X+Z \geq Y_{d}$

the production scale of the park is limited by resources. The vector of regional initial input of given product structure and scale is denoted as $U_{X}=\left(E^{T}, U^{T}\right)^{T}$. Of which, $E$ is the energy vector of $s$ dimension, and $U$ is other resource input vector of $r-s$ dimension. Whether energy or non-energy resource, and whether local natural resources or external resources, the supply quantity within certain period is restricted, and is denoted as $U_{c}$, hence we obtain the second constraint condition:

$U \leq U_{c}$

Assume that the production scale of the park is not limited by technology, thus there is no explicit production scale limit in the model. In production, the by-product substitutes for initial resource input, which equivalently increases the resource supply quantity. On condition that the resource is restricted and the production scale is not restricted, it is in favor of the expansion of production scale. However, the usage amount of by-product is limited by its output, thus we obtain the third constraint condition $d_{\max } \leq b$, i.e.:

$\left(\mathbf{B}^{T}-\mathbf{D}\right) X \geq 0$

This condition is too strict to the production scale. On condition that the initial input resource is permissible, we can consume the resource to improve the production scale, and generally, the actual usage amount of by-product is $d \leq d_{\max }$. Therefore, Formula (7) is unnecessary constraint condition, which can be broadened as:

$d \leq b$

Next, we will build the optimization mode through discussing the objective function, control variable.

\subsection{Optimization Model}

The final source of the park economic income is the final consumption determined by its output level (leave out the 
product price), which is guaranteed by the first constraint condition Formula (5).

Under the objective condition that the resource is limited, the by-product generated from the production process of the product of the park not only replenishes the resource supply of the park, but also reduces the resource price. Therefore, one of the bases of determining the optimization objective is that the usage amount of by-product is the maximum.

Assume that $d$ is the actual usage amount of by-product, for the given by-product utilization scheme, it is the function of gross output in the park, and the corresponding vector of regional emission is $P_{X} \triangleq b-d=\mathbf{B}^{T} X-d$.

The pollution level to environment due to industrial emission is measured according to the hazards of different emissions, and the units of measurement are different, such as air pollution, water pollution and soil pollution, etc. In order to make the pollution generated from all by-product emissions have comparability, it is necessary to determine the comparable coefficients of different pollutants to unify the measurement criterion of regional pollution level, for example, we can take the domain value of 100 credit system or equivalent [0-1] domain value. For clarity, we take the measurement pollution index of [0-1] domain value in this model, and assume that the environment pollution of different emissions has additive property, namely that the gross contamination generated from a kind of emission is the simple accumulation of unit emission pollution.

For the given by-product (pollutant), the degree of contamination generated from unit emission is determined. If we denote the pollution index of unit emission of No. $j$ byproduct as $\delta_{j}, \delta=\left(\delta_{1}, \delta_{2}, \cdots, \delta_{m}\right)$, under the given parameter and assumed condition, the general pollution index of the region is $p=\delta P_{X}=\delta\left[\mathbf{B}^{T} X-d\right]$, and the optimized objective is $\min p$.

Divide the gross output vector into two parts: $X=X_{T}+X_{D}$, $X_{T}$ and $X_{D}$ are respectively generated with the initial input and recycled material in Table $\mathbf{1}$ as raw material. Now the initial input vector is $u=\mathbf{T} X_{T}$ the use vector of by-product is $d=\mathbf{D} X_{D}$, and the objective function is $p=\delta\left(\mathbf{B}^{T} X-\mathbf{D} X_{D}\right)$.

Based on Formula (2), the optimization model is obtained through combining the constraint conditions Formulae (5), (6) and (8):

$$
\begin{aligned}
& \min _{X, X_{D}} p=\delta\left(\mathbf{B}^{T} X-\mathbf{D} X_{D}\right) \\
& \text { s.t. }\left\{\begin{array}{l}
(\mathbf{I}-\mathbf{A}) X+Z \geq Y_{d} \\
\mathbf{T}\left(X-X_{D}\right) \leq U_{c} \\
\mathbf{D} X_{D} \leq \mathbf{B}^{T} X \\
X \geq X_{D} \geq 0 \\
X>0
\end{array}\right.
\end{aligned}
$$

\section{MODEL OPTIMAL SOLUTION}

The incompleteness of model (9) mainly lies in its constraint conditions, involving the existence of solution and trivial solution
$Z$ in the constraint condition of Formula (5) is the vector of product imported from the outside of the park, mainly used for regulating the supply of this product of this park, but it does not embody more effect in the objective function or other constraint conditions, and it may cause trivial optimal solution, for example, as long as we take $Z=Y_{d}$, $X^{*}$ will be the optimal solution besides the fifth constraint condition.

There are two solutions to this problem. The first solution is to correct $Z$ into net import (import-export), $Y$ is the final consumption of the region, on the condition that the market demand of the region $Y_{d}$ and the net import $Z_{d}$ are given, the first constraint condition of Formula (9) becomes (I-A) $X \geq Y_{d}-Z_{d}$, thus we obtain the relatively complete planning model:

$$
\begin{aligned}
& \min _{X, X_{D}} p=\delta\left(\mathbf{B}^{T} X-\mathbf{D} X_{D}\right) \\
& \text { s.t. }\left\{\begin{array}{l}
(\mathbf{I}-\mathbf{A}) X \geq Y_{d}-Z_{d} \\
\mathbf{T}\left(X-X_{D}\right) \leq U_{c} \\
\mathbf{D} X_{D} \leq \mathbf{B}^{T} X \\
X \geq X_{D} \geq 0 \\
X>0
\end{array}\right)
\end{aligned}
$$

However, the contradiction conditions (I-A) $X \geq Y_{d}-Z_{d}$ and $\mathbf{T} X_{T} \leq U_{c}$ in the formula may cause no solution. Thus it should be solved through improving the initial resource supply vector $U_{c}$ and the by-product usage amount .

In fact, $\mathbf{T} X_{T} \leq U_{c}$ is equivalent to $\mathbf{T} X \leq U_{c}+\mathbf{T} X_{D}$, of which, $\mathbf{T} X_{D}$ is the vector of initial resource equivalent to by-product, and $\mathbf{T X}$ is the whole input including byproduct. Therefore, as long as $U_{c}+\mathbf{T} X_{D}$ is big enough, the intersection of $(\mathbf{I}-\mathbf{A}) X \geq Y_{d}-Z_{d} \quad$ and $\mathbf{T} X_{T} \leq U_{c} \quad$ is nonempty.

The second solution is to set the "import total products as less as possible" mechanism in the model. In fact, the product import $Z$ is only used for compensating the insufficient product supply of the park, and its criterion is "as less as possible on condition of meeting the primary demand". We can set "punishment mechanism" to hit the mark, namely take the imported product vector $Z$ as the control variable, and put punishment factor to the objective function:

$$
\begin{aligned}
& \min _{X, X_{D}} p=\delta\left(\mathbf{B}^{T} X-\mathbf{D} X_{D}\right)+M Z \\
& \text { s.t. }\left\{\begin{array}{l}
(\mathbf{I}-\mathbf{A}) X+Z \geq Y_{d} \\
\mathbf{T}\left(X-X_{D}\right) \leq U_{c} \\
\mathbf{D} X_{D} \leq \mathbf{B}^{T} X \\
X \geq X_{D} \geq 0 \\
X>0
\end{array}\right.
\end{aligned}
$$


Of which, the element of row vector $M$ of $n$ dimension is equivalent to certain positive number which is big enough. Different from Formula (10), here we can avoid the contradiction of the first two constraint conditions through adjusting export vector $Z$.

On condition of not taking technical capacity into account, based on the natural conditions of the region, make $\mathbf{D} X_{D} \leq \mathbf{B}^{T} X$ as nonempty with appropriate industrial arrangement.

We can observe $d_{\max }=\mathbf{D}\left(X_{D}+X_{T}\right) \geq d$, thus the constraint condition of Formula (8) is looser than that of Formula (7), and guarantees $p=\delta\left(\mathbf{B}^{T} X-\mathbf{D} X_{D}\right) \geq 0$. Obviously, the necessary and sufficient condition that Formula (7) is equivalent to Formula (8) is $\mathbf{D} X_{T}=0$, now:

$\sum_{k=1}^{n} d_{j k} x_{T k}=0,(j \in \underline{m})$

If $\sum_{k=1}^{n} t_{i k} x_{T k}=0,(i \in \underline{r})$, it indicates that the production inputs of all products use by-product or intermediate input. On the above assumed condition, the production in this park does not consume any natural resource of this park and any extraneous natural resource, which is an impossible park product structure, thus $i \in \underline{r}$ exists, making $\sum_{k=1}^{n} t_{i k} x_{T k}>0$. Of which, certain $k \in \underline{n}$ must exist to make $x_{T k}>0$. Because $D$ is nonnegative matrix (each element is nonnegative), from Formula (12) we can see $d_{j k}=0,(j \in \underline{m})$, now $x_{D k}=0$; conversely, if certain $d_{j k}>0$, then $x_{T k}=0$. Thus $x_{T k} x_{D k}=0,(k \in \underline{n})$, and the necessary and sufficient condition that Formula (7) is equivalent to Formula (8) is that the process that the by-product manufactured from the production of the product of the park is used for the initial input can be separated, i.e. $X_{T}^{\prime} X_{D}=0$.

The fourth constraint condition $X \geq X_{D} \geq 0$ means $X_{T}=X-X_{D} \geq 0$, and condition $X>0$ shows that the region manufactures the products in Table $\mathbf{1}$, all these meet the essential requirements in normal circumstances.

In conclusion, through appropriate adjustment, there is optimal solution in model (9), and there is better optimization operability.

\section{CONCLUSION}

The structure of the model built in this paper is simple, with preferable operability, which can be taken as the supplementary mean and science basis of industrial structure planning of industrial park. However the function and resource endowment of industrial park are different in thousands of ways, and the model parameters should be determined according to the specific characteristics of the park, as well as that the effectiveness and adaptation of the model need further inspection. Finally, the application and further research for this model are described simply as follows:
1) Model (9) and improved (10) or (11) are the framework planning models with regional product structure as control variable. It is suggested that the survey should be carried out according to the industrial layout of the park, and determine the model variable and parameter with the functional orientation and resource endowment of the park as basis. For the determination of parameter, many aspects of actual data should be considered comprehensively;

2) The application of vector $\delta$ of contamination coefficient in the objective function is the important basis for Model (9) to stand. If this parameter does not exist technically or cannot be determined, it is suggested that other equivalent coefficient should be used, and the corresponding objective should be changed into other corresponding objective from the minimum environment pollution;

3) The model assumes that "the emission is the waste of energy", and substitutes the indirect objective "minimum emission" for the direct objective "improve the energy utilization efficiency", thus it does not embody or use energy value conversion coefficient in Table 1. It is suggested to consider the direct energy efficiency objective in the further research;

4) For the energy optimization, the comprehensive benefit of the park should be concerned, and it is allround. This model only contains environmental benefit (the pollution index is the minimum) in the objective function, and indirectly contains income benefit in constraint condition $\left(Y \geq Y_{d}\right)$. It is suggested to do meticulous work in further research, considering price, cost and other regional comprehensive economic benefits, as well as commuting traffic, environmental influence of grabbing natural resources and other comprehensive social benefits of the park;

5) In view of the limitation of data resource and research deadline, the optimization model built in this paper does not give actual application case study, which needs further research.

\section{CONFLICT OF INTEREST}

The authors confirm that this article content has no conflict of interest.

\section{ACKNOWLEDGEMENTS}

Project supported by national natural science foundation of China (71071022).

\section{REFERENCES}

[1] Wu, Q.; Wu, C. The research on the development model of China eco-industrial park. Chin. J. Popul. Resour. Environ., 2012, 22(7), 60-66.

[2] Liu, H.; Yang, T. The research on the influence factors of energy, economy, environment coordination development of industrial park. Enterprise Econ., 2013, 2013(3), 140-143. 
[3] Song, X.; Shen, J. Industrial planning modeling of eco-industrial park and NSGA-II-IFD algorithm based on multi-objective optimization. Chin. J. Popul. Resour. Environ., 2014, 24(9), 68-74.

[4] Li X.; Peng N.; Zhou Y. International sustainable consumption practice and policy implication. Chin. J. Popul. Resour. Environ., 2014, 24(5), 46-50.

[5] Han, Q.; Liu, Z. Total quantity control-based bi-level planning model of energy distribution of industry area. Chin. J. Manag. Sci., 2013, 21(2), 168-174.
[6] MacKenzie, I.A.; Hanley, N.; Kornienko, T. Using contests to allocate pollution rights. Energy Policy 2009, 37(7), 2798-2806.

[7] Ohshita, S. Target allocation methodology for China's provinces: Energy intensity in the 12th five-year plan. California: Lawren Berkeley National Laboratory, 2011.

[8] Lin, G.; Wang, Z. The estimation of embodied energy of China merchandise trade export - based on input-output method. $J$. Beijing Inst. Petrochem. Technol., 2013, 21(1), 58-62.

(C) Xin and Zhenquan; Licensee Bentham Open.

This is an open access article licensed under the terms of the Creative Commons Attribution Non-Commercial License (http://creativecommons.org/licenses/by-nc/3.0/) which permits unrestricted, non-commercial use, distribution and reproduction in any medium, provided the work is properly cited. 